\title{
Estudio de los pueblos mágicos en México: el caso del municipio de Chignahuapan, Puebla
}

\section{Study of the magical towns in Mexico: The case of the municipality of Chignahuapan, Puebla}

Alfredo Perez-Paredes; Verónica Juárez-Fonseca²;

Amado Torralba-Flores ${ }^{3}$

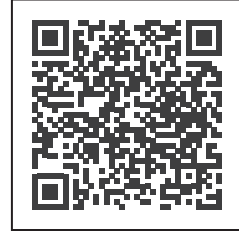

\section{Palabras clave:}

Chignahuapan; cultura; economía; municipio; pueblos mágicos; turismo.

Artículo de investigación

Fecha de recepción:

16 de abril de 2021

Fecha de aprobación:

14 de diciembre de 2021

Fecha de publicación:

23 de diciembre de 2021

Esta publicación se encuentra bajo licencia:

Creative Commons ReconocimientoNoComercial-SinObraDerivada 4.0 Internacional

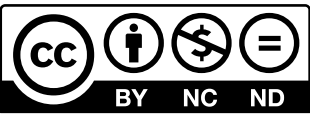

\section{Resumen}

Problemática: en México, la denominación de pueblos se realizó con los propósitos de fortalecer a las comunidades y de mejorar la competitividad de las empresas, las condiciones laborales de sus trabajadores y la calidad de vida de la comunidad. Sin embargo, al paso de los años se observa que esto no ocurre. Objetivos: estudiar al municipio de Chignahuapan desde la mirada socioeconómica y cultural del turismo y contribuir con propuestas al fortalecimiento de la comunidad. Materiales y métodos: el tipo de investigación fue cuantitativa, toda vez que se aplicaron cuatro tipos de encuestas, así como la indagación documental, que confluyeron para la construcción del marco conceptual. Resultados: Ios principales resultados encontrados por parte del gobierno municipal indican que a causa de la denominación de pueblo cuentan con recursos adicionales. En lo correspondiente a los visitantes, se encontró que el 100 \% de estos son mexicanos y

1 Licenciado en Administración de Empresas y doctor en Administración Pública, Facultad de Administración, Benemérita Universidad Autónoma de Puebla, México. alfredo.perez@correo.buap.mx, ORCID: https://orcid. org/0000-0001-8766-5766

2 Contadora pública y auditora. Doctora en Investigación e Innovación Educativa, Facultad de Administración, Benemérita Universidad Autónoma de Puebla, México. titosfonseca@yahoo.com.mx, ORCID: https://orcid.org/00000002-0562-1063

3 Contadora pública y auditora. Doctora en Investigación e Innovación Educativa, Facultad de Administración, Benemérita Universidad Autónoma de Puebla, México. admon_buap_at@yahoo.com.mx, ORCID: https://orcid. org/0000-0003-3802-050 


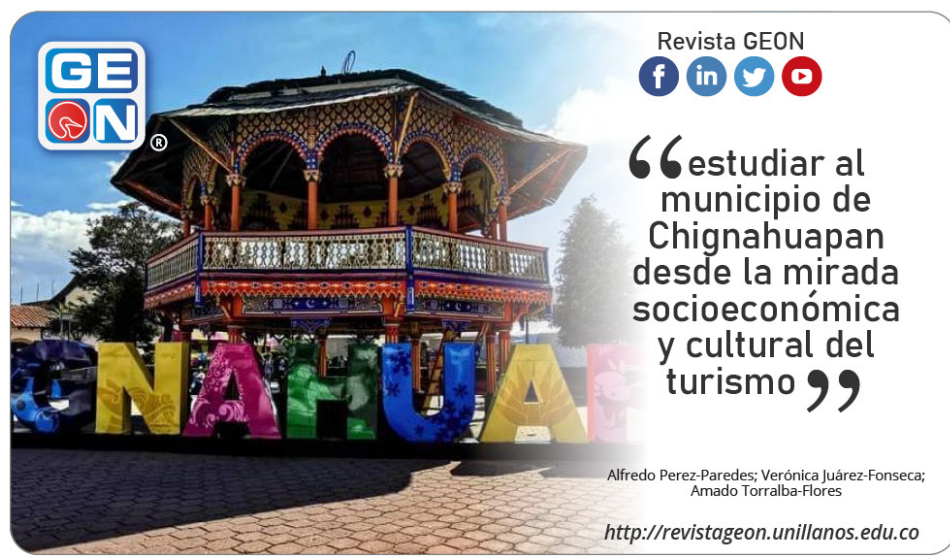

que las calificaciones por el servicio recibido no son las mejores. En cuanto a los ciudadanos, estos consideran que la denominación de pueblo no ha incrementado el turismo y tampoco ha disminuido las actividades productivas tradicionales. Discusión: de los múltiples resultados explorados se encontró que, en el tema de la economía, la ciudadanía considera que el turismo en efecto ha contribuido al crecimiento del empleo, aunque esta contribución es temporal y no ha mejorado la calidad de vida de los habitantes. Conclusiones: se concluye que hay una necesidad de darle mayor visibilidad a la comunidad para incrementar la afluencia turística y con ello contribuir a mejorar el nivel de vida de los habitantes de tan importante lugar. Contribución/ originalidad: la presente contribución se considera original, toda vez que no se han encontrado investigaciones con estas características sobre las implicaciones de la denominación de pueblo mágico para la comunidad de Chignahuapan.

Palabras clave: Chignahuapan; cultura; economía; municipio; pueblos mágicos; turismo.

Códigos JEL: Z320 Turismo y desarrollo

\section{Abstract}

Problem: In Mexico, the denomination of towns took place in order to strength the local communities and in with the attention of improving the competitive- 
Cómo citar este artículo / Toreference this article:

Perez-Paredes, A., Juárez-Fonseca, V., \& Torralba-Flores, A. (2021). Estudio de los pueblos mágicos en México: el caso del municipio de Chignahuapan, Puebla. Revista GEON (Gestión, Organizaciones Y Negocios), 8(2), e-472. https://doi. org/10.22579/23463910.472 ness of some companies, including their working conditions and rights for their human resources as well as the quality of the community life itself. However, as the years go by, there has not been such improvement. Objectives: To study the town of Chignahuapan and its municipality from a socio-economic and cultural perspective of its tourism in order to contribute with some proposals to strengthen the community opportunities. Methods: This is quantitative research, since four types of surveys were applied, as well as documentary research, which converged for the construction of the conceptual framework. Findings/Results: The main results found by the municipal government indicate that, because of its town name, the community of Chignahuapan gets to receive some additional resources and benefits. Respecting the visitors, it was found that a hundred per cent of these are actually Mexican people and the rates they gave to the community services mentioned before are just not the best. In the other hand though, the local citizens consider that the denomination of town has not significantly increased the tourist inflow and neither has it decreased the town's traditional productive activities. Discussion: According to the multiple results explored in the present research, it was found that most citizens consider that tourism has indeed contributed to the growth of employment which has as well improved the community economy although this contribution is temporary, therefore it has not improved the quality of the community's life. Conclusions: There is indeed a need for the community to be seen and recognized with a greater visibility so that they can increase the tourist influx in an effort to contribute and improve the quality of the way they live in such an important place. Contribution/Originality: This contribution is considered original research, since there is not any other research with the characteristics presented here regarding the implications of the name of magical town which is a touristic brand for the Chignahuapan community.

Keywords: Chignahuapan; culture; economy; magical towns; municipality; tourism.

JEL codes: LEL: Z320 Tourism and Development 


\section{Introducción}

En México, el turismo es muy importante para la economía del país, ya que es generador de divisas y fuentes de empleo, además de que contribuye al desarrollo económico de algunas localidades que cuentan con un inventario turístico representado por su arquitectura, tradiciones y paisajes peculiares. Derivado de esto, en 2001 la Secretaria de Turismo (Sectur, 2019) formalizó el programa Pueblos Mágicos.

Dado lo anterior, en el estado de Puebla, México, al municipio de Chignahuapan se le asignó la categoría de pueblo mágico, debido a que en algunas de sus actividades económicas destacan, por ejemplo, la producción y comercialización de esferas navideñas, altamente demandadas a nivel internacional, o la técnica de vidrio soplado, con la que se forman figuras que tienen gran demanda a nivel nacional. A esto se suman sus paisajes pintorescos y representativos, como son el Salto de Quetzalapan, lugar idóneo para practicar el rapel - lanzarse desde la tirolesa. También destacan la Basílica de la Inmaculada Concepción así como su gastronomía, representada por la barbacoa de hoyo y sus dulces de jamoncillo.

Derivado de lo anterior, la presente investigación estudia las diversas características del municipio de Chignahuapan para diseñar propuestas que coadyuven al fortalecimiento del turismo. Por lo tanto, la búsqueda exploratoria de los antecedentes del pueblo, su medio físico, población, cultura, servicios públicos y econo- mía permitieron reconocer que la organización endógena del municipio muestra una atención importante de gestión de políticas públicas.

Adicionalmente, en la investigación se enmarcan los elementos teóricos derivados del trabajo heurístico así como metodológico que guio a la investigación, seguido del desarrollo histórico de la región, para posteriormente mencionar el ideario que caracteriza al municipio. Finalmente, se destacan los hallazgos que llevaron a la reflexión y a las propuestas que promuevan el fortalecimiento endógeno y global del municipio.

\section{Contexto teórico}

Antes de abordar el concepto de municipio, es preciso centrarse en el tema de la gobernanza como parte de las políticas públicas, debido a que este incide en varios sectores de estudio, como son el educativo, el económico y el cultural, por mencionar algunos; en este último se presenta una estrecha vinculación con el tema del municipio, pues el hito pretende fortalecer las acciones de un gobierno a partir de la capacidad de ser administrado eficaz y eficientemente. El acercamiento conceptual de gobernanza deriva en lo siguiente: "La gobernanza se convierte en un enfoque con sentido político [...] permite la inclusión de los actores políticos y sociales en la toma de decisiones y como parte de su estructura de gobierno, lo cual permite una gestión política" (Jiménez Hernández, 2019).

En este sentido, la dicotomía gobernanza y municipio se une en una es- 
trecha relación que intenta alcanzar un bienestar económico y social a través de gestiones perennes de cada entidad, o bien a un municipio.

A decir de este, el concepto de municipio proviene del latín mumus, que significa cargas u obligaciones, tareas u oficios. Otra acepción proviene del verbo capere, que quiere decir hacerse cargo de algo o de ciertas cosas. Estos términos se destacan como palabras compuestas. De este modo, la Ley Orgánica Municipal del estado de Puebla (LOMEP), en su capítulo I, establece que un municipio es libre y con derecho público, que está conformado por un territorio a cargo de un gobierno de elección popular que atiende las necesidades colectivas de la población y que la conduce a participar para la promoción integral de la comunidad. Este, a su vez, goza de personalidad jurídica y de patrimonio propio (artículo $2^{\circ}$ y $3^{\circ}$, respectivamente) (Honorable Congreso del Estado Libre y Soberano de Puebla, 2001).

Otra perspectiva similar es la que ofrece el acervo de la biblioteca virtual del Instituto de Investigaciones Jurídicas de la Universidad Autónoma de México, la cual consiste en que el municipio se concibe como

[...] una persona jurídica de derecho público, compuesta por un grupo social humano interrelacionado por razones de vecindad al estar asentado permanentemente en un territorio dado, con un gobierno autónomo propio y sometido a un orden jurídico específico, con el fin de mantener el orden público, prestar servicios públicos indispensables para satisfacer necesidades elementales. (Martínez Gil, 2009)

Conviene ahora enfocar la concepción de municipio desde una visión cultural como eje central del tema de investigación; es decir, desde el tema turístico sostenible. De acuerdo con la Secretaría de Turismo (Sectur), el municipio es determinante para la vinculación con los procesos de responsabilidad social, la innovación y la actividad económica matizada de cultura en cada país, estado o municipio, por lo que las políticas públicas intentan rescatar la identidad de cada región, para que los destinos turísticos promuevan la sostenibilidad a corto, mediano y largo plazo con visión social, económica y ambiental, que permee en cada región.

Lo antes mencionado ha servido como base para que, en el caso de México, se pusiera en marcha a comienzos del siglo XXI un programa denominado Pueblos Mágicos, con la intención de implementar las buenas prácticas del turismo sostenible y así garantizar el mínimo impacto sobre los recursos naturales. Además, la calidad del servicio se convirtió en un rubro de producto turístico y con ello alcanzar el mejor desempeño socioeconómico, para beneficiar a los turistas y, en consecuencia, a la comunidad en particular.

A decir de lo anterior, el municipio objeto de estudio, que goza de personalidad jurídica con visión turística sostenible, presenta ciertas caracte- 
rísticas que lo hacen único, como se estudiará más adelante. Continuando con la revisión conceptual, es preciso destacar antes el tema del turismo, no solo como una actividad de recreación, sino también como una estrategia de políticas públicas encaminadas a promover un territorio de beneficio social y económico. Derivado de esta reflexión, Pérez-Ramírez y Antolín-Espinosa (2016) señalan que el turismo proviene de una actividad turística que consolida a la comunidad, promueve el mercado y lo beneficia, por lo que adquiere un papel protagónico. Todo ello con la delineación de políticas públicas.

Finalmente, la Organización Mundial del Turismo (OMT, 2018) considera al tema del turismo sostenible como prácticas de gestión aplicadas a todas las formas de turismo y que deben conducirse bajo los principios de sustentabilidad y al cuidado del medio ambiente, económico y sociocultural, para que, en este caso, el municipio de Chignahuapan permita tener un equilibrio entre el medio ambiente y la disminución de la pobreza.

Por lo anterior, las políticas públicas que en México han sido delineadas para promover algunos municipios como "pueblos mágicos" con identidad propia fungen como de impulsoras para una nueva forma de organización a largo plazo. Pero, entonces, ¿qué es un pueblo mágico?, ¿qué lo hace ser único? A continuación, se presentan algunas reflexiones que intentarán dar respuesta a estos cuestionamientos.

\section{Pueblos mágicos}

El programa Pueblos Mágicos es una estrategia de fortalecimiento del sector turístico sostenible. Se concibe que un pueblo mágico sirve para enaltecer niveles de bienestar y acrecentar el empleo, y permite, además, incrementar la economía de alguna región en particular aprovechando sus recursos, cultura, infraestructura, servicios que ofrece, así como atractivos naturales y de innovación, preservando las tradiciones ancestrales. Asimismo, intenta salvaguardar y proteger las riquezas culturales de la región, conservar su pasado indígena y arquitectónico, preservar el legado histórico y las tradiciones de cada municipio en particular, para brindar de esta manera una oferta turística diversificada. Dicho programa estampa el valor agregado a través de los atributos, leyendas, historias y demás expresiones socioculturales para generar un mayor atractivo para los turistas.

Las características esenciales que identifican a un pueblo mágico son sus casas multicolores, sus paisajes agradables que dan sociego, su variedad gastronómica y las artesanías propias, así como la creatividad e innovación de sus habitantes; todo ello lo hace único (Sectur, 2019). A esto se suma el propósito del pueblo mágico, que significa generar otros beneficios a los recursos naturales y culturales de la región para favorecer su economía y desarrollo, disminuir los niveles de pobreza, mejorar la calidad de vida de sus habitantes y brindar bienestar en la prestación de servicios a los visitantes. Además de estas 
políticas públicas (Gobierno Municipal de Chignahuapan, 2018), existen los siguientes objetivos importantes: a) diseñar e implementar una oferta turística orientada a un mercado interesado en aspectos naturales, culturales e históricos; b) innovar con el diseño de nuevos productos históricos aprovechando las particularidades de las comunidades; c) generar mayores beneficios a los integrantes de las localidades; d) implementar servicios turísticos de calidad; e) capacitar al capital humano para beneficio de las empresas turísticas; f) estimular las inversiones por parte del sector gubernamental y empresarial; g) generar una oferta turística sólida en México; h) consolidar destinos turísticos en desarrollo; i) reorientar el turismo en las localidades consolidadas; y j) promover el desarrollo sostenible a través del turismo.

Como se observa, los objetivos están orientados a la aspiración de capacidades que son inalcanzables para algunos municipios, dado que esta pretensión deriva de proyectos a largo plazo. Por ejemplo, la construcción de programas estructurados en todos los niveles: económico, político, sociocultural y físico, que confluyan en el ordenamiento turístico de cada territorio, así como su interacción con otros sujetos (residentes y visitantes). La intención es la conservación del patrimonio, el aumento de la economía y la satisfacción de servicios (hoteles, agencia de viaje, restaurantes, entre otros) y el mejoramiento de la calidad de vida (Pérez-Ramírez \& Antolín-Espinosa, 2016). En todo caso, los objetivos se traducen en desafíos para cada localidad. De este modo, se tiene que, en el caso de México, la asignación a esta nueva política de significación municipal se gestó como se describe a continuación.

En México, el origen del programa Pueblos Mágicos se dio en el año 2001, durante el período presidencial de Vicente Fox Quezada. El nombramiento de los primeros pueblos mágicos tuvo lugar en los estados de Hidalgo (pueblo mágico Huasca de Campo) y de San Luis Potosí (pueblo mágico Real de (atorce), los cuales fueron el parteaguas de una nueva identidad regional. Posteriormente y hasta el momento, se han incluido otros estados en el citado programa, para un total de 121 municipios (Sectur, 2019). Entre estos, el municipio de Chignahuapan en particular ha sido el promotor hacia la mejora en la calidad de vida de sus habitantes.

\section{Materiales y métodos}

Los criterios de selección del diseño metodológico dependieron de los factores externos que incidieron en esta investigación. Por tanto, se destaca que la presente investigación, de tipo mixto (cualitativo y cuantitativo), así como el alcance exploratorio significaron el desarrollo de un diseño no experimental, transeccional y descriptivo, que propició una guía sistematizada sin transgredir las variables que de esta emanaron. Se observó además que el contexto estudiado confluyó en interacciones importantes.

Con respecto a los factores externos, se destaca que, de acuerdo con lo 
planteado por Cadena-Iñiguez et al. (2017), en la investigación cuantitativa, son los instrumentos los que guían a la investigación, debido a que los datos se acumulan y la información se generaliza. Esto permite cuantificar la información obtenida en resultados más certeros, por lo que la muestra representativa hace inferencia en una población definida.

Con respecto a la población objeto de estudio, la elección grupal dependió del contexto generalizado; esto es, autoridades representativas de la región, tipo de empresas (comerciales y de servicio), así como la población aleatoria de los visitantes. Lo anterior se desglosa como se muestra a continuación:

a). autoridades locales;

b). población en general del pueblo mágico;

c). empresarios y comerciantes;

d). turistas o visitantes.

De este modo, los informantes clave correspondieron a 10 autoridades. Respecto a la muestra, se tuvieron en cuenta 60 empresarios, 223 ciudadanos y 166 visitantes. En cuanto a la temporalidad de aplicación, esta tuvo lugar en el mes de diciembre de 2019y a principios de enero de 2020. De esta población objeto de estudio, pudo obtenerse una relación e intercambio dialéctico como ocurre en cada contexto en donde se aplica el trabajo de campo (Gurdián-Fernández, 2007).

Asimismo, se realizó una revisión de fuentes secundarias que coadyuvaron a construir el marco teórico-conceptual, para generar los fundamentos de cada uno de los apartados presentados. En cuanto a la técnica aplicada, la entrevista estructurada fue determinante. Con respecto al instrumento, se diseñaron cuatro cuestionarios que fueron aplicados en diferentes sujetos pertenecientes al pueblo mágico en estudio.

\section{Resultados}

Los estudios realizados dan cuenta de una glosa con datos y reflexiones categorizados como se muestra a continuación.

\section{Autoridades poblacionales}

La edad de los ciudadanos en el rubro de cargos públicos va de los 25 a los 59 años. De estos, el 50 \% corresponde al sexo masculino y el $50 \%$ al sexo femenino. Se destaca que tan solo el $30 \%$ tiene estudios de licenciatura. Cabe mencionar que, para la obtención de información, se consideró, además, la participación y colaboración del presidente municipal, así como de regidores, directores y jefes de departamento.

Al preguntarles cuál es la principal actividad económica del municipio de Chignahuapan, el $80 \%$ considera que es el turismo y el $20 \%$ restante, la agricultura. Un $50 \%$ coincide en que el ingreso más importante que tiene la comunidad es el turismo, que genera más fuentes de empleo. También se mencionó que el porcentaje de inversión pública para el turismo es más del $50 \%$ del presupuesto. 
Por otra parte, el $90 \%$ considera que se han generado actividades adicionales al turismo, pero ello no ha implicado el abandono de las tradiciones; asimismo, un 40 \% consideran que sí mejoraron los servicios públicos y el $60 \%$ restante señala que la infraestructura ha mejorado.

ambién se preguntó si los turistas influenciaban el comportamiento de la población y se encontró que, con respecto a los cambios en la cultura, las costumbres y las tradiciones, un $40 \%$ de los entrevistados considera que hubo cambios totales, un $30 \%$ piensa que estos cambios fueron parciales y el $30 \%$ restante afirma que no hubo cambios. En cuanto a la organización de la comunidad, se determinó que el $20 \%$ considera que fue un cambio total, el $40 \%$ piensa que fue un cambio parcial y el $40 \%$ afirma que no hubo cambios. En lo que respecta a la economía de las familias, se identificó que el $50 \%$ considera que hubo cambios totales, el $30 \%$ piensa que estos cambios fueron parciales y el $20 \%$ afirma que no hubo cambios.

También se encontró que, en cuanto a las autoridades, el servicio que ofrecen a la comunidad radica en que los ciudadanos reciben un trato especial, tanto en instancias federales, como en estatales; es deir al referirse a los proyectos especiales, un 50 \% respondió que casi siempre; tratándose de temas de financiamiento, un $50 \%$ respondió que siempre y en cuanto al tema de recursos materiales y en especie, un 33,33\% respondió afirmativamente.
Para finalizar, se encontró que un $50 \%$ de los encuestados considera que el desarrollo del pueblo con la operación del programa es bueno y el 50 \% restante piensa que es excelente. A grandes rasgos se observa que hay una buena percepción por parte de las autoridades en cuanto a la denominación de pueblo mágico de Chignahuapan.

\section{Visitantes}

En lo que concierne a este rubro, los resultados dan cuenta de que, para este sector en particular, la intencionalidad de conocer un pueblo mágico es primigenia. Por tanto, a manera de censo, se tiene que la edad de los visitantes está entre los 15 años y los 64 años. Además, el 53 \% de los visitantes corresponde a sexo masculino y el $47 \%$, a sexo femenino. Otro dato importante consiste en que el $31 \%$ de los visitantes cuenta con estudios de licenciatura y posgrado.

Por otra parte, se encontró que el $100 \%$ de los encuestados son mexicanos, lo que llama la atención, debido a que se pensó que con la denominación de "pueblo mágico" el municipio gozaría de mayor presencia internacional. En cuanto a los visitantes nacionales, se constató que estos provienen de estados como Puebla, Tlaxcala, Veracruz, Ciudad de México, Estado de México e Hidalgo.

Además, se encontró que en el $41 \%$ de los encuestados la preferencia de alojamiento es los hoteles; en el $9 \%$ es la Boutique Hotel; en el $15 \%$ es las casas de pensión; en el 16\% es las 
casas de huéspedes; en el $9 \%$ es los hostales y el resto prefiere otro tipo de hospedaje. De toda esta parcela se tiene que tan solo el 23 \% lleva a cabo la reservación; el resto considera que no es necesario anticipar su estancia, lo que significa que el municipio aún no ha alcanzado un gran impacto turístico. En cuanto a la experiencia de hospedaje, se obtuvo que el $30 \%$ la califica como "excelente", el $34 \%$ como "buena", el 26 \% como "regular", el $3 \%$ como "mala" y solo el $1 \%$ como "pésima".

A estos hallazgos se suma que los principales productos que se consumen corresponden a las artesanías, con un $38 \%$, seguidas de productos gastronómicos, con un $34 \%$, y finalmente con productos varios, que representan el $28 \%$ restante. En cuanto a la dicotomía calidad y precio, significó que el 52 \% de los visitantes encontró que los productos y servicios antes mencionados son de fácil acceso. En cuanto al pago de estos servios, se encontró que un 30 \% decidió realizar sus pagos de forma electronica y el $18 \%$ restante consideró que las diversas formas de pago no eran relevantes. Se destaca esta forma de pagos de servicio que ocurre en un pueblo mágico; debe promoverse el consumo local y el pago tradicional, como una manera de preservarlos.

Asimismo, cuando se evaluó la hospitalidad y el trato recibido por parte de los visitantes, se encontró que solo el $13 \%$ los considera "excelentes", el $9 \%$ los califica como "buenos" y el $77 \%$ piensa que son "regulares"; el $1 \%$ restante los considera como "malos" o "pésimos". En cuanto a la atención recibida, el $49 \%$ opina que es "excelente", el $31 \%$ piensa que es "buena", el $18 \%$ considera que es "regular" y el $2 \%$ afirma que es "mala" o "pésima". También se encontró que, en relación con la solución de problemas que les atañe, el $24 \%$ considera que esta es "excelente", el $21 \%$ la califica como "buena", el 36 \% afirma que es "regular" y el $19 \%$ restante piensa que es "mala" o "pésima". Por último, en lo que corresponde a la recepción de sugerencias o recomendaciones de parte de los lugareños, se determinó que el $27 \%$ la evalúa como "excelente", el 33 \% piensa que es "buena", el $33 \%$ afirma que es "regular", el $6 \%$ considera que es mala y el $1 \%$ piensa que es "pésima". Quizá esta tendencia se deba al estrato sociocultural.

En cuanto al uso del transporte público, se identificó que el $46 \%$ utiliza los autobuses, el $25 \%$ ocupa el servicio de taxis, el $13 \%$ utiliza trenes y el $16 \%$ usa las bicicletas públicas. Se encontró, además, que solo el $11 \%$ y el $27 \%$ evaluaron como "excelente" y "bueno" el servicio recibido, respectivamente.

Adicionalmente, el $49 \%$ de los visitantes califica su estancia como "excelente", considerando que se cumplieron sus expectativas; el $31 \%$ piensa que esta es "buena", el $14 \%$ la califica como "regular", el $3 \%$ indica que esta es "mala" y el $3 \%$ restante afirma que es "pésima". Otra parcela de la población investigada, correspondiente al $49 \%$, valora la convivencia como "excelente"; un $32 \%$ indica que es "buena"; un $15 \%$ señala que esta 
es "regular" y el $4 \%$ restante afirma que es "mala". En relación con la atención personalizada, el $45 \%$ la califica como "excelente"; el 36 \% piensa que es "buena"; el $15 \%$ considera que es "regular" y el $4 \%$ restante afirma que es "pésima". Para finalizar este apartado, se encontró que el $49 \%$ de los encuestados califica las experiencias vividas como "excelentes", el $37 \%$ piensa que son "buenas" y el 14 \% restante las considera como "regulares".

Como nota complementaria, se tiene que el $29 \%$ de los visitantes califica su estancia en cuanto a la calidad de los servicios ofrecidos como "excelente", mientras que el $48 \%$ y el $23 \%$ consideran que es "buena" y "regular", respectivamente. Por último, a los visitantes se les preguntó si recomendarían a alguien visitar el pueblo mágico y el 98 \% respondió que "sí lo haría" y solamente el 2 \% contestó que "no", lo que representa, en términos generales y con base en los resultados obtenidos, que los servicios ofrecidos a los visitantes son buenos. Esto significa que aún existen áreas de servicio por atender para mejorar el servicio al cliente.

\section{Ciudadanos y lugareños del municipio}

La edad de los ciudadanos de Chignahuapan va de los 15 a los 64 años; el $55 \%$ de estos es de sexo masculino y el $45 \%$ restante es de sexo femenino. Se destaca que el $41 \%$ de esta población tiene estudios de licenciatura y posgrado.

Con respecto a la principal actividad económica de la comunidad, el $43 \%$ considera que es la agricultura. Además, se preguntó sobre qué actividad económica genera más ingresos y se encontró que el 52 \% asegura que el turismo es la más relevante en este punto. También se indago qué tanto interés hay para emplearse en actividades asociadas al turismo y se encontró que la actividad que más interés genera es la del comercio, a la que le sigue la industria y, por último, la pesca.

Por otra parte, el $57 \%$ de los ciudadanos considera que no se han desarrollado otras actividades económicas asociadas al turismo; el 66 \% afirma que no se han dejado de realizar las actividades tradicionales por las del turismo; por último, el $84 \%$ señala que la situación económica para la comunidad por ser considerada como pueblo mágico ha sido muy buena, mientras que un 54 \% piensa que esta es buena y que beneficia a toda la comunidad.

También se indagó por qué tipo de inversionistas tiene el sector turismo y se encontró que el 86 \% es internos y solo el $14 \%$ es externos. Adicionalmente, un $64 \%$ considera que algunas veces estas empresas producen algún tipo de beneficio. Un 96 \% de los encuestados asegura que gracias a la denominación de "pueblo mágico" han llegado más turistas al municipio de Chignahuapan y el 94 \% indica que el período de mayores visitas es el que va de septiembre a diciembre.

De igual manera se indagó si los turistas eran respetuosos de las tradiciones locales durante las visitas a la 
comunidad y se encontró que el $96 \%$ piensa que sí lo son. También se preguntó si ocasionaban algún tipo de problema y el $67 \%$ de los encuestados responde que no los provocan. Por último, el 62 \% afirma que la influencia de los turistas es positiva.

Finalmente, se preguntó sobre los recursos que los ciudadanos consideran más importantes y se encontró que el 41 \% piensa que el recurso más importante es sus monumentos históricos, a los que le siguen los recursos forestales, con el $40 \%$, y los recursos hidráulicos, con el $19 \%$ de los encuestados. También se preguntó cuáles eran los recursos más valorados por la población y el 45 \% respondió que son los recursos forestales. En cuanto a si los cambios de los recursos en los últimos tres años han sido buenos o malos, el $75 \%$ de los ciudadanos considera que han sido malos y el $38 \%$ piensa que el factor que ha motivado los cambios en los recursos naturales han sido los cambios climáticos. Por último, el $71 \%$ afirma que no existe un programa de conservación de recursos naturales.

\section{Empresarios}

En este rubro se identificó que la edad de los empresarios está entre los 15 y los 69 años. De esta parcela, el 52 \% es de sexo masculino y el $48 \%$ es de sexo femenino. Se destaca que el $37 \%$ tiene estudios de licenciatura y posgrado, lo que significa que la escasa formación profesional es un tema que requiere atención como agenda en las políticas públicas.

En cuanto a las entidades enfocadas en el turismo, se encontró que estas representan el $46 \%$ y están a la par de las comerciales, por lo que la competitividad se ciñe más en estas que en las de orden agrícola e industrial (3\%, cada una). Además, se destaca que el $88 \%$ es empresas micro, que tan solo el $7 \%$ corresponde al sector pequeño y que el $3 \%$ escasamente es de tipo mediano. Esta clasificación sitúa a este sector en una competencia parcial, por lo que se abre la brecha de las oportunidades de crecimiento empresarial.

Por otra parte, el rango de ingresos per cápita mensual se desglosa de la siguiente manera: de 1000 a 5000, con un $36 \%$; de 5001,00 a 10.000,00, con un $39 \%$; de 10.001 a 15.000, con un $11 \%$; y más de 15.000, con el $14 \%$. La asimetría de ingresos provoca desigualdades de oportunidad para el crecimiento económico en las familias, con tendencia a que con el paso del tiempo esta brecha se abra aún más.

En cuanto al tema de la categoría de "pueblo mágico", si bien el 57 \% de la comunidad considera un aumento en sus ingresos, existe un $43 \%$ en contraposición que afirma que esto derivó en disminución de sus ingresos, proclive a pérdidas. Esta situación coloca en desventajas a las empresas minoritarias (misceláneas), a pesar de los retos atendidos por el sector gubernamental. Entonces ia qué se debe la causa de la disminución de ingresos de un sector más desfavorecido?

Algunas respuestas surgidas permitieron observar que las oportunidades de crecimiento y desarrollo empresarial se deben al tipo de enfoque 
de atención a la sociedad; es decir, la creación de empresas y proyectos que beneficien a ciertos sectores. Sin embargo, las oportunidades que se siguen apreciando son selectivas. En cuanto a la creación de nuevas empresas de turismo, el $49 \%$ señala que son locales, el $17 \%$ indica que son franquicias, el $9 \%$ resalta que son foráneas y el $25 \%$ afirma que no se han creado empresas. También se preguntó si habían otras instancias que hubieran realizado algún proyecto que impulse la economía de la región y se obtuvo que el 50 \% de estos corresponde a infraestructura, el $17 \%$ a innovación empresarial y desarrollo de productos turísticos, el $22 \%$ a proyectos con enfoque a la comercialización y tecnología y, por último, el $11 \%$ a otros.

\section{Discusión}

La presente disertación constituye una reflexión que no solo intenta vislumbrar las necesidades o áreas de oportunidad de crecimiento del municipio objeto de estudio, sino que además permite contrastar con algunos de los principios enfocados al turismo que los organismos internacionales delinean en relación con el tema de turno. De acuerdo con la Organización para la Cooperación y el Desarrollo Económicos (OCDE, 2017), el turismo representa un valor por encima de la economía, pues este es un agente generador de empleo, por mencionar un ejemplo. Sin embargo, el impulso del turismo en este municipio de Chignahuapan aún no es equiparable a la economía de acuerdo con su contexto, pues la dis- crepancia radica en los hallazgos que se describen a continuación.

De los múltiples hallazgos explorados, hay uno que merece mayor significación y que corresponde al tema de la economía. Desde esta óptica, la ciudadanía considera que el turismo, en efecto, ha contribuido al crecimiento del empleo; sin embargo, este es débil, dada la temporalidad, por lo que el $91 \%$ coincide en que los beneficios se presentan durante períodos discontinuos y no son garantía de una economía sustancial que coadyuve a sus servicios básicos, como alimentación, salud y otros de necesidad importante.

Respecto a la evaluación de la calidad de vida de los habitantes, se obtuvo que por encima del $50 \%$ coinciden en que esta es "regular". A pesar de esta valoración que la sociedad emite y aún con la insignia que tiene el municipio, existe otra perspectiva de que la actividad empresarial es una fuente importante, tal como se menciona a continuación.

Los efectos económicos en Chignahuapan, sin duda, han provocado una ruptura entre clases sociales. No significa la oposición o crítica a la categoría de este municipio, pero sí a la atención del diseño de estrategias que promuevan y aceleren a las clases menos favorecidas, dado que más del $50 \%$ de la población considera que aún no se ha visto beneficiada del "pueblo mágico".

A lo largo de más de una década, los diseños de acciones que buscan alternar las políticas de crecimiento 
económico y turístico aún son incipientes. Para este caso en particular, evaluar el programa Pueblos Mágicos permitió dar más luz crítica para la implementación de políticas públicas que promuevan, por un lado, la formación profesional universitaria y, por otro, la participación de egresados. Asimismo, el apoyo a los microempresarios sirvió para que ya no se vuelvan solamente sujetos de estadísticas desfavorecidas, sino de estudios cualitativos que revaloren el papel de su desempeño.

\section{Conclusiones}

A partir del objetivo señalado en la investigación, consideramos que el programa Pueblos Mágicos implantado en la localidad de Chignahuapan, si bien cuenta con alto potencial turístico, la captación internacional aún es laxa, por lo que deben privilegiarse nuevas estrategias para fortalecer el turismo del municipio.

Respecto al tema que subyace a la evaluación del impacto del programa Pueblos Mágicos en términos de generación de empleos directos-indirectos, unidades e ingresos económicos como resultado de la actividad turística, puede considerarse de atención importante la generación de nuevas empresas y fuentes de empleos que permitan mejorar el nivel de vida.

Por otra parte, se analizó el grado de participación y beneficio que obtuvo la población con la operación del programa y se encontró una buena apreciación tanto de los ciudadanos como de los empresarios encuesta- dos, aunque parece ser, de acuerdo con los resultados obtenidos, que no perciben un beneficio económico que mejore su calidad de vida. Esto es algo sobre lo que debe trabajarse conjuntamente entre las autoridades y todos los actores que contribuyen a la actividad del turismo.

Pudo identificarse que, con respecto al tema de desarrollo social, se clasifican en tres niveles significativos, como son el social, el cultural y la infraestructura. En el primero se observa que los ciudadanos reconocen la importancia de la denominación y cómo esta ha beneficiado las actividades del turismo; por ello puede valorarse como positivo en este sentido. Posteriormente, en el tema cultural, no se percibe una gran variación después del nombramiento de pueblo mágico, debido a que antes de que recibieran esta insignia, los temas culturales ya estaban asentados y esto es un área de oportunidad. Por último, se visualiza que en el tema de la infraestructura sí se perciben beneficios debido a las inversiones tanto públicas como privadas que se han realizado y que han beneficiado a la comunidad.

Sin duda alguna, la perspectiva de autoridades locales, población, turistas y empresarios permite identificar cuáles son los beneficios que se han generado en la localidad de Chignahuapan y así incrementar el tema del turismo; sin embargo, se identificó que solamente la visitan turistas cercanos al estado de Puebla, lo que pone de manifiesto la necesidad de darle mayor visibilidad a la comunidad para incrementar la afluencia tu- 
rística y con ello contribuir a mejorar el nivel de vida de los habitantes de tan importante lugar.

Se espera que con la presente investigación y propuestas que se realizan puedan desarrollarse posteriores trabajos que permitan mejorar el desarrollo de estas localidades llamadas "pueblos mágicos".

\section{Información complementaria.}

\section{Agradecimientos.}

Contribuciones de autoría: Alfredo Pérez-Paredes y Verónica Juárez-Fonseca elaboraron el marco contextual y conceptual, Amado Torralba-Flores, los resultados, y entre los tres autores el resto del artículo.

Conflictos de interés: no existen conflictos de intereses.

Financiamiento: la investigación fue autofinanciada.

Material suplementario: no aplica.

\section{Referencias}

Cadena-Iñiguez, P., Rendón-Medel, R., Aguilar-Ávila, J., Salinas-Cruz, E., De la Cruz-Morales, F.R. \& Sangerman-Jarquin, D. M. (2017). Métodos cuantitativos, métodos cualitativos o su combinación en la investigación: un acercamiento en las ciencias sociales. Revista Mexicana de Ciencias Agrícolas, 8(7), 1603-1617. https://bit.ly/3qhsmrT

Gobierno Municipal de Chignahuapan. (2018). Plan de Desarrollo Municipal 2018-2021. https://bit.ly/3qkiGgi

Gurdián-Fernandez, A. (2007). El Paradigma Cuaitativo en la Investigación
Socioeducativa. Coordinación educativa y cultural centroamericana (CECC), Agencia Española de Cooperación internacional (AECI). Editorial Educativo Regional. https://bit.ly/3yYfUkN

Jiménez Hernández., G. (2019). Un acercamiento conceptual sobre gobernanza desde el enfoque de la política pública. Revista Isceem, 14(3), 81-86. https://bit.ly/3pdSwwh

Honorable Congreso del Estado Libre y Soberano de Puebla. (2001). Ley Orgánica Municipal 2016. Por la cual expide el Honorable Congreso del Estado de Puebla LIX Legis/atura. https://bit. ly/3208B9K

Martínez Gil, P. (2009). El municipio, la ciudad y el urbanismo. En Régimen jurídico del urbanismo. Memoria del Primer Congreso de Derecho Administrativo Mexicano. Universidad Nacional Autónoma de México. https://bit. ly/3peOluF

Organización Mundial del Turismo (OMT). (2018). Acerca de la OMT. https://bit. ly/3yMPHFK

Organización para la Cooperación y el Desarrollo Económicos (OCDE). (2017). Estudio de la Política Turística de México. Resumen ejecutivo, evaluación y recomendaciones. OCDE, mejores políticas para una vida mejor. https://bit. ly/3H19kwE

Pérez-Ramírez, C. A. \& Antolín-Espinosa, D. I. (2016). Programa pueblos mágicos y desarrollo local: actores, dimensiones y perspectivas en El Oro, México. Revista de Alimentación Contemporánea y Desarrollo Regional, 25(47), 218242. https://bit.ly/3pdwHwM

Secretaría de Turismo de México (Sectur). (2019). Chignahuapan, Puebla. https://bit.ly/3ml30YR 